\title{
Anna GĘSICKa
}

Université Nicolas Copernic, Torun

iD 0000-0001-6394-2562

\section{Entre jeu et nostalgie Les topoï courtois dans Les Trois Aveugles de Compiègne de Jean Ott}

Between play and nostalgia. The courtly topoï in Les Trois Aveugles de Compiègne by Jean Ott

AbSTRACT: The subject of this paper is an analysis of Jean Ott's theater play Les Trois Aveugles de Compiègne. In his adaptation of a 13th-century famed "fabliau" Jean Ott (1878-1935), a lesser-known French author, offers its receiver an interesting intertextual play. In a manner that is both parodic and nostalgic he appeals to the courtly topoi, treating it as a signpost that defines the development of action and the semantic scope of his work.

KeY Words: Jean Ott, courtly topos, parody, nostalgia

Dans son adaptation théâtrale d'un fabliau médiéval portant le même titre, Les Trois Aveugles de Compiègne, Jean Ott, auteur oublié du début du $\mathrm{XX}^{\mathrm{e}}$ siècle, propose à son récepteur un jeu intertextuel intéressant. Parmi les différents niveaux de ce jeu, se distingue une façon ambivalente dont l'auteur se réfère à la topique courtoise. Puisque cet élément est totalement absent dans l'hypotexte, son omniprésence dans l'hypertexte attire d'autant plus l'attention du lecteur/ spectateur. L'ordre de ma démarche embrassera trois étapes: d'abord le rappel des principaux topoï de la fin'amor, puis la présentation de la silhouette de Jean Ott, enfin l'analyse des échos de l'idéologie et du code courtois repérables dans son texte'.

${ }^{1}$ La question de la forme de l'adaptation, de la réécriture d'un fabliau dans un cadre théâtral précis, n'est pas l'objet de mon intérêt dans cet article. A cette problématique, entre autres, sera consacré mon livre Jeux transtextuels. Les fabliaux médiévaux sous la plume de Jean Ott (XX siècle), dont la rédaction progresse peu à peu. 
Comme on le sait bien, le terme «amour courtois» a été créé à la fin du $\mathrm{XIX}^{\mathrm{e}}$ siècle par Gaston Paris, pour désigner un type nouveau de relation amoureuse observable dans l'univers littéraire, aussi bien dans la poésie lyrique des troubadours (la fin'amor) que, un peu plus tard, dans le roman. La courtoisie est un terme plus large qui désigne un mode de vie raffiné, se caractérisant par l'élégance, la mesure, les bonnes manières, la caritas. L'amour courtois serait la réalisation suprême de ces principes dans le cadre littéraire et signifierait une manière d'aimer réservée aux élus, aux âmes d'élite, différente de celle du vulgaire ${ }^{2}$.

L'idéologie de la fin'amor s'organise selon un certain code, calqué sur les rapports et rites féodaux, s'exprimant dans l'emploi de topoï précis que l'on peut retrouver dans tous les poèmes courtois ${ }^{3}$. Michel Zink écrit :

[...] un poème [...] doit passer, pour produire une impression sur le lecteur, par les modes d'expression conventionnels de l'affectivité. Le jeu poétique, pour les troubadours, consiste donc non pas à rechercher l'originalité, mais à se conformer le plus possible à un modèle idéal, tout en y introduisant $d u$ jeu par des décalages, des innovations, des raffinements menus et, plus essentiellement, par l'infinité des variantes combinatoires entre les motifs convenus.

ZINK, 1983: 136

L'essentiel en est que pour le poète courtois, aimer veut dire chanter. L'amour courtois est associé au printemps, à la lumière, et s'exprime essentiellement par le chant. C'est une passion qui anoblit le poète, le force à chanter et qui est en même temps la source principale de son inspiration. Au centre de l'amour courtois figure la Dame, occupant la place du suzerain. Elle est de haut parage, belle, parfaite, distante et inaccessible, exceptionnelle et la plus noble. C'est la Dame sans Merci, froide, exigeante et parfois cruelle dans ses exigences. Devant elle - un Amant Parfait, amoureux et fidèle, idolâtrant sa Dame et supportant avec patience toutes les souffrances que celle-ci lui impose. L'Amant exerce à l'égard de sa Dame un service d'amour copié sur le service féodal. Il exécute tous les ordres de sa Dame, satisfait tous ses désirs, fait des exploits guerriers en son honneur, tout en observant la règle principale du code courtois qui est la discrétion absolue sur la personne de l'amante, à cause du mari (qui est de préférence vieux et jaloux, l'antithèse de l'amant courtois, toujours jeune, joven) et des gens jaloux qui peuvent nuire à leur amour (les lauzengiers). C'est pour cela qu'on utilise dans cette poésie les senhals (pseudonymes). La souffrance quotidienne de l'Amant est paradoxalement pour lui une source de joie - le joy, terme

${ }^{2}$ Cf. p. ex. Frappier (1973: 1-31), Duby (1999), Marrou (1971: 150-163), Zink, Wind (1992: 334-342).

${ }^{3}$ Cf. p. ex. Siciliano (1934: 313-336), Marrou (1971: 125-143), Marty-Dufaut (2002 : 13-33), ZINK (1983 : 128-153). 
difficile à traduire, veut dire un mélange de joie et de tristesse et un élan positif fourni par ce sentiment. Il souffre quand même, surtout à cause de la jalousie et de la crainte de perdre les faveurs de sa Dame. Le cœur de l'Amant est enfermé dans une véritable prison d'amour; la dame en garde la clé. Il veut s'en libérer, mais n'arrive pas à le faire - ou ne le veut pas vraiment? Il faut souligner le rôle du regard-miroir dans la naissance du sentiment: l'amant voit une belle dame, se reflète dans ses beaux yeux (aspect narcissique de cette poésie) et l'amour éclateJour et nuit, l'Amant est brûlé par le désir. C'est dans le code courtois le topos le plus important. Par sa nature, le désir, sur lequel est fondé chaque amour, veut être assouvi, ce qui peut entraîner sa diminution ou disparition. L'amour est donc une tension: un conflit entre le désir d'être assouvi et la crainte de cet assouvissement trop rapide qui signifie la fin du désir, donc de l'amour... C'est pour cela que l'amour courtois est de nature adultère. L'amant désire non seulement le cœur, mais aussi - surtout? - le corps blanc de sa Dame, dont la description ne donne jamais de détails concrets sauf des superlatifs de nature générale. C'est un amour érotique, en dépit des apparences parfois trompeuses. L'amour courtois doit être récompensé. La récompense finale (le guerredon) est le moteur du service amoureux. Avant, la Dame octroie à son serviteur un regard favorable, un anneau, un baiser; puis l'asag - épreuve finale (sur ce point, les avis des critiques partagés), au lit, consistant «à permettre de tener, abrassar, baizar, manejar, pourvu que ces caresses ne conduisent pas au coït proprement dit. À moins que la dame n'en décide autrement» (Crorx, 1999: 48)

Pour être heureux, l'amour courtois doit être réciproque. Si la dame rejette l'amour que lui porte l'amant parfait, si elle tarde trop à réaliser ses promesses, lui, il devient Amant Martyr. Il veut partir - c'est le topos du congé d'amour. Avant de partir, il laisse à sa Dame cruelle son cœur en don, enchâssé comme une relique. Il fait souvent son testament ou compose sa propre épitaphe. Il déclare qu'il arrête de chanter c'est-à-dire d'aimer. Il est sûr qu'il mourra d'amour.

Jean Ott (1878-1935), dans sa vie professionnelle, a débuté comme un Ingénieur ordinaire des Ponts et Chaussées, pour finir comme Chef du Service technique du Métropolitain de Paris, contribuant à la construction de plusieurs lignes. Pendant la Grande Guerre, comme capitaine au $5^{\text {ème }}$ génie, il a participé, entre autres, à la bataille de la Marne et à la bataille de Verdun (Suquet, 1935 : 215-216; Berton, Ossadzow, Filloles, 1998 : 213).

Pourtant, Jean Ott est aussi un auteur aujourd'hui méconnu; un poète et auteur dramatique fécond et talentueux assez fécond et bien doué. Malheureusement, il n'est pas mentionné dans les histoires de la littérature, même celles du théâtre. Jean Ott a ouvré dans le domaine de la poésie

${ }^{4}$ Charles Baladier fournit plus de précisions : les deux amants «nus et enlacés atteignent l'acmé de la continence en interdisant à leurs caresses les plus sensuelles de franchir la limite de l'orgasme» (BALADIER, 1999: 165-166). 
scientifique (L'Effort des races, Les volontés) (GHIL, 2008: 119, note 13), mais surtout du théâtre : il est auteur de nombreuses pièces brèves inspirées de légendes médiévales et d'adaptations théâtrales de cinq fabliaux du $\mathrm{XIII}{ }^{\mathrm{e}}$ siècle.

L'adaptation des Trois Aveugles de Compiègne de Jean $\mathrm{Ott}^{5}$ transforme l'histoire de son célèbre hypotexte portant le même titre, et introduit des personnages nouveaux. Le fabliau médiéval est construit autour d'un méchant tour joué par un jeune clerc à trois mendiants aveugles. Le clerc feint de leur donner une pièce de monnaie; chacun pense qu'un autre l'a prise. Les aveugles se permettent donc une belle soirée dans une taverne, puis, bien sûr, n'ont pas de quoi payer, d'où résulte une suite de séquences amusantes. La pièce de Jean Ott, dont l'action se passe aussi au XIII ${ }^{\mathrm{e}}$ siècle, commence à la taverne, par un dialogue entre Hélène, la fille du tavernier, et un jeune Chevalier qui lui fait la cour sans succès. La scène suivante, c'est l'arrivée des trois aveugles qui, dans l'adaptation de Jean Ott, sont artistes-ménestrels. Chacun porte un nom symbolique, renvoyant au sens qui est son guide-conducteur remplaçant la vue. Le Chevalier veut leur jouer un drôle de tour. C'est la réécriture de la séquence du fabliau avec la pièce de monnaie donnée à on ne sait pas qui (car chacun des aveugles pense qu'un autre l'a reçue). Le Chevalier n'assiste pas au développement de l'intrigue. Les aveugles se décident à rester dans la taverne, en demandant au tavernier du meilleur vin, puis n'ont de quoi payer. C'est Hélène qui, comprenant qu'ils sont victimes de son prétendant, leur vient au secours et calme son père qui s'accorde même à ce qu'ils passent la nuit chez eux, dans un coin modeste («le coin des sceaux et des balais », 16). Ici s'arrête toute fidélité à l'hypotexte, Jean Ott remplaçant la scène à l'église par une intrigue «médiévalisante» tout à fait nouvelle. Vient donc une longue scène «courtoise» où Hélène, telle une grande dame entourée d'une cour d'admirateurs, mène avec ses invités une conversation érudite et riche en divers accents intertextuels. Puis, elle préside à la séance de trois chants à caractère philosophique exécutés par les ménestrels. La pièce se termine par une drôle de vengeance morale faite sur le Chevalier, qui devient la victime humiliée d'un complot raffiné préparé par les trois aveugles.

Dans sa pièce, Jean Ott insiste sur la dimension élitaire et anoblissante de l'amour courtois, signalant son aspect érotique d'une façon plutôt camouflée. Une phrase-clé, qui revient plusieurs fois en fonction d'un signe renvoyant à la culture médiévale, est l'accusation ironique d'Hélène formulée à l'adresse du Chevalier: "Vous n'êtes pas courtois ». Tout le dialogue introductif entre Hélène et son prétendant participe du génie courtois, traité par Jean Ott d'une part sur un ton satirico-parodique, mais de l'autre, avec une note de nostalgie. Celle-ci est sensible dans le personnage d'Hélène, qui essaie d'enrichir sa vie monotone

${ }^{5}$ Édition citée: Отт, 1930-1932: 1-34. Les chiffres entre parenthèses renvoient à la pagination. 
d'accents excitants, puisant dans le modèle courtois. Dans le dialogue qui suit, nous retrouverons le cadre parodié de la fin'amor, avec les topoï reconnaissables : exploits dans le service d'amour, cœur insensible et cœur ardent, clef d'amour, chant d'amour, lumière, baiser et désir, et finalement une sorte d'asag... :

\section{HÉLÈNE}

Que ferais-je de vous qui n'êtes pas courtois?

$$
\text { LE CHEVALIER }
$$

Ogier l'est-il ?

HÉLÈNE

Il sait du moins par quels exploits

On fascine les cœurs, comme Amadis de Gaules,

Fussent-ils plus gelés que les glaciers des pôles!

LE CHEVALIER

Moi, je sais faire rire et je suis beau garçon.

HÉLÈNE

Mais vous n'entendez rien à la douce chanson

Qui berce tendrement le cœur des amoureuses

Et remplit du soleil l'ennui des heures creuses.

LE CHEVALIER

Ogier la chante-t-il?

HÉLÈNE

Il sait la suggérer.

Vous, vous riez, alors qu'on désire pleurer!

Vous êtes beau garçon, chevalier, oui, peut-être,

Mais je suis veuve et comme telle ai pu connaître

Ce que devient bientôt le joli séducteur,

Quand on lui met au doigt la clef de notre cœur!

LE CHEVALIER

Votre premier mari vous fut-il si funeste?

HÉLÈNE

Du tout! puisqu'il me fait priser ce qui me reste!

Pourriez-vous, tous deux seuls, en des lieux retirés,

Sans un baiser, pendant cinq ans, près de moi, vivre?

LE CHEVALIER

Pendant cinq ans, auprès d'un corps qui vous enivre?... HÉLĖNE

Vous ne le feriez pas? Vous n'êtes pas courtois!

LE CHEVALIER

Courtois ! toujours courtois !... qu'est-ce que ce patois?

Comme nous voyons, non seulement la topique lyrique de la fin'amor est manifestement familière à Hélène, mais généralement celle-ci fait preuve, ce 
qui peut étonner, d'une culture livresque solide, connaissant les titres-modèles des romans courtois. Dans les répliques éloquentes et érudites, elle évoque non seulement un roman célèbre Amadis de Gaule, dont la première version du XIII ${ }^{e}$ siècle est attribuée à João Lobeira (ResınA, 2014 : 401), mais aussi un chef d'œuvre de la littérature courtoise française:

\section{HÉLÈNE}

C'est celui, cher ami, du Roman à la Rose.

Apprenez-en trois mille vers, cela s'impose !

LE CHEVALIER

Des vers! laissons ces jeux aux ménestrels manchots.

Cela semble même être un dialogue entre deux parties du Roman de la Rose: la réplique d'Hélène se réfère à la partie de Guillaume de Lorris exposant les subtilités du code courtois, alors que celle du Chevalier, à l'accent mis sur les «jeux» verbaux, renvoie, fût-ce inconsciemment (suite à un instinct cynique), à Jean de Meun démasquant l'essence mensongère de l'amour courtois. Dans la suite de ce dialogue, où le reproche «vous n'êtes pas courtois » est redit à trois reprises, Hélène évoque la forêt de Brocéliande (une forêt mythique citée dans plusieurs textes appartenant à la légende arthurienne), alors que le Chevalier répète sur le même ton sceptique: «Tout cela c'est chanson» (7).

Hélène, romantique, estime les hommes selon leur sensibilité et leur culture courtoises. Dans sa critique du Chevalier, où nous retrouvons d'ailleurs aussi une allusion à la matière de Bretagne et à l'innocence des sentiments nobles, c'est ainsi qu'elle le caractérise devant les trois aveugles:

Ce chevalier est un méchant homme, un cœur vide,

Qui méprise l'amour courtois et les romans.

Tout s'enchaîne et se tient parmi les sentiments !

Je crains l'esprit en qui toute féerie est morte.

$[\ldots]$

Cette âme basse, au pur amour ne saurait croire!

(16-17)

Jugé selon le même critère, l'autre prétendant s'avère plus heureux. Hélène, en Dame courtoise, mais non Sans Merci, apprécie ses efforts. On y aura reconnu aussi les topoï courtois de la relation dame-amant parfait (avec un certain renversement des rôles), du service amoureux et du secret obligatoire: «Ogier fait, près de moi, le stage le plus droit, / Je suis sa dame en courtoisie, il est mon roi ; / Mais je vous parle là de choses bien secrètes! » (17).

Dans la scène suivante, dont le témoin caché est à nouveau le Chevalier revenu, les mots-clés se rattachent également à l'idéologie et au code courtois. 
D'abord, le baiser. A l'intention du Chevalier inconscient, les aveugles jouent une petite comédie. Ils discourent d'un baiser hypothétique de la veuve qui «est un morceau du roi », ce qui signifie que «son baiser doit-être unique»; c'est un don spécial pour celui qui le mérite (19). Puis, à nouveau le secret - et un lauzengier à craindre: «Ce n'est pas nous qui trahirons pour un infâme / Le doux secret que nos oreilles ont surpris » (22). Ensuite, l'idéalisation d'Hélène fait penser aux éloges troubadouresques, aussi enflammés qu’imprécis, et au désir inassouvi des Amants Parfaits: «Son corps présentera tous les trésors du monde !», «On sentira près d'elle un désir de mourir...», «Elle se glissera, la divine maîtresse, / Comme une griserie au cœur...» (21).

Il y a d'autres allusions littéraires. Dans le fragment qui suit, nous retrouverons des renvois aux célèbres fables et romans courtois. Le nom de Chrétien de Troyes frappe par son absence, même si le lecteur reconnait tout de suite les allusions littéraires évoquant ses œuvres. Par contre, le mot sémantiquement central semble être le «rite»:

\section{LE TOUCHER}

Qui sait s'il passerait l'épreuve de l'écu? [...]

\section{L'OUIE}

Il est ignare !...

Connaît-il le Saint-Graal que comme un nom bizarre?

L'ODORAT

Ce bellâtre sait-il seulement passer l'eau

Sur le pont d'une épée ainsi que Lancelot?

Remettrait-il la flamme à la lampe fragile

Ainsi qu'aux temps romains fit l'enchanteur Virgile ${ }^{6}$ ?

La dame est délicate et, de l'amour courtois,

Ogier connaît le rite, et sur le bout des doigts.

Il sait ce qu'est la coupe, et le sceptre, et le casque.

Dans le dialogue entre le Chevalier venu à l'embuscade et le Toucher, revient le mot de passe proposé par les aveugles dans le cadre de l'intrigue dirigée contre le Chevalier: "Les mots: 'Je suis ARTUS!' qu'il faut dire à l'huis » (23). C'est une citation parodiée provenant de La chanson de Roland ou de Roncevaux $d u X I I^{e}$ siècle (« Je suis Artus dont l'en a tant parlé», 837 : 209). On y évoque le personnage du roi légendaire, en fonction d'un signe central de l'univers courtois :

\section{LE TOUCHER au judas}

Qui donc es-tu qui frappe là?

\footnotetext{
${ }^{6}$ Sur la figure complexe de Virgile dans la littérature médiévale cf. LefÈvre (1992).
} 
LA VOIX $d u$ chevalier au dehors.

Je suis Artus!

\section{LE TOUCHER}

Des Artus, il en est sur terre par centaines...

Es-tu le roi des provinces armoricaines?

VOIX DU CHEVALIER

Heu... c'est-à-dire oui... Oui, je suis le roi des lieux

Que vous dites.

Tout le «doux spectacle » (23), préparé minutieusement par les aveugles pour humilier définitivement le Chevalier, est basé sur le concept de l'«initiation dans les amours courtoises» (23). Le Chevalier, ayant déclaré à priori sa disposition à « passer les épreuves » (31), y est en effet soumis, toutes les chicanes comprises. Dans cette scène de vengeance, dont la belle Hélène est absente, le contexte courtois est complètement tourné en dérision. On ligote les mains du Chevalier (signe qu'il est «dompté»), on lui explique que «souffrir est nécessaire. / La souffrance est l'état qu'il faut pour s'enivrer!», on lui bande les yeux, car ils «empêchent de voir au seuil du merveilleux», on lui entrave les pieds au nom d'un «rite», on l'appelle «Roi». Finalement, on l'équipe d'attributs reconnaissables: "Il te manque la coupe, et le sceptre, et le casque». Ceux-ci sont faits d'une "poële à frire, toute noircie ${ }^{7} »$, «d'un affreux balai», d'une "espèce de pot de chambre», avec l'information que c'est un «casque guerrier baisé par Esclarmonde» $(31-33)^{8}$.

Conscient de la valeur des signes au Moyen Age, Jean Ott y fait allusion expressis verbis, dressant devant le récepteur une vision resémantisée d'épiphanie du Graal: «De la coupe de feu les signes sortiront. / Au nom de Belzébuth, je te consacre au front! (Il lui barbouille la figure avec du noir de fumée)» (33). L'épreuve consiste à traverser un pont fait d'une planche disposée sur deux tables, au-dessous duquel est placé «un baquet plein d'eau savonneuse» (33). Dans la dernière scène parodique, avec encore une fois le célèbre exploit de Lancelot au centre, la rhétorique courtoise sert de prétexte efficace de vengeance:

\section{LE TOUCHER}

Laisse au vent flotter tes oriflammes!

(il lui fait bouffer sa chemise au dehors de ses chausses).

Et fais deux pas vers ce baiser que tu réclames.

(le chevalier met le pied sur la planche qui s'effondre,

il tombe dans l'eau savonneuse du baquet avec un

bruit épouvantable. Accourent avec des lumières, le

${ }^{7}$ Les italiques indiquent la didascalie.

${ }^{8}$ Esclarmonde est, entre autres, l'hérö̈ne d'une chanson de geste du XIII' siècle, Esclarmonde, faisant partie du cycle de Huon de Bordeaux (cf. Ruelle, 1992). 


\section{Tavernier et sa fille).}

HÉLÈNE

Dieu que se passe-t-il ?... Est-ce vous, chevalier?

LE CHEVALIER

Le pont de Lancelot n'aurait pas dû plier!

L'adaptation du fabliau médiéval faite par Jean Ott joue visiblement sur la parodie de l'idéologie et de la topique de l'amour courtois. Ainsi, l'auteur du $\mathrm{XX}^{\mathrm{e}}$ siècle s'apparente aux auteurs médiévaux, coutumiers de multiples formes de parodie et d'auto-parodie ${ }^{9}$. Pourtant, elle trahit aussi une grande part de nostalgie. Premièrement, semble-t-il, ceci se voit dans la personne d'Hélène, simple fille du tavernier, qui dans son fond intérieur se nourrit de cette idéologie et la recherche dans sa modeste vie. La nostalgie d'un grand amour, pur et gratuit, participe d'un rêve universel, trouvant des expressions littéraires à toutes les époques. Et deuxièmement, en lisant la pièce de Jean Ott, nous sentons également sa propre nostalgie de l'époque passée et de ses belles lettres. L'abondance de références intertextuelles appropriées et sophistiquées en est la preuve.

\section{Bibliographie}

Baladier, Charles 1999 : Éros au Moyen Age. Amour, désir et «delectatio morosa ». Paris, Cerf. Berton, Claude; Ossadzow, Alexandre; Filloles, Christiane 1998: Fulgence Bienvenüe et la construction du métropolitain de Paris. Paris, Presses des Ponts.

Croix, Arnaud de la 1999: L'érotisme au Moyen Age. Le corps, le désir et l'amour. Paris, Tallander.

Duby, Georges 1999: «À propos de l'amour que l'on dit courtois». In : IDEm : Féodalité. Paris, Gallimard, p. 1415-1420.

FraPPIER, Jean 1973: Amour courtois et Table ronde. Genève, Droz.

GHIL, René 2008: De la poésie scientifique et autres essais. Textes choisis, présentés et annotés par Jean-Pierre BaвiLlot. Grenoble, Ellug.

Jouvenel, Emile 1928: «Réponse de M. Emile Jouvenel à M. Georges Seguin». Bulletin de l'Académie du Var, p. 27-28, http://www.academieduvar.org/bulletinacad/1928.pdf, accès : le $26 \mathrm{~V} 2010$.

La chanson de Roland ou de Roncevaux du XII siècle, 1837. Francisque Xavier Michel (éd.). Paris, Silvestre Librairie.

Lefèvre, Sylvie 1992: «Virgile au Moyen Age». In: Dictionnaire des lettres françaises. Le Moyen Age. Geneviève Hasenohr, Michel Zink (éds). Paris, Fayard, p. 1481-1483.

Madureira, Margarida; Carreto, Carlos Clamote; Morais, Ana Paiva (éds.) 2016: Parodies courtoises, parodies de la courtoisie. Paris, Classiques Garnier.

Marrou, Henri-Irénée 1971 : Les troubadours. Paris, Seuil.

9 Cf. Marty-Dufaut (2002: 171-198), Madureira, Carreto, Morais (2016). 
Marty-Defaut, Josy 2002: L'amour au Moyen Age. De l'amour courtois aux jeux licencieux. Autres Temps.

Отт, Jean 1930-1932: Cinq fabliaux mis en pièces. Paris, Imprimerie O. Dousset.

PARMentier, Florian 1914: La littérature et l'époque. Histoire contemporaine des lettres françaises de 1885 à nos jours. Paris, Eugène Figuière, http://www.archive.org/details/lalittraturel00parmuoft, accès: le $20 \mathrm{~V} 2010$.

Resina, Joan Ramon 2014: «Iberian Peninsula». In: The Encyclopedia of the Novel. Vol. 1. Peter Melville Logan et all. (éds.). Willey-Blackwell, p. 399-413.

RuElle, Pierre 1992: «Huon de Bordeaux (Cycle de)». In : Dictionnaire des lettres françaises. Le Moyen Age. Geneviève Hasenohr, Michel Zink (éds). Paris, Fayard, p. 703-706.

Siciliano, Italo 1934 : François Villon et les thèmes poétiques du Moyen Age. Paris, Librairie Armand Colin.

SuQuet, Louis 1935 : «Jean Ott - notice nécrologique». Bulletin du PCM, p. 215-216,

http://www.unipef.org/sites/unipef/files/PCM/avant1973/1935 05_Integrale.pdf, accès: le $16 \mathrm{~V}$ 2015.

ZINK, Michel 1983: «Troubadours et trouvères». In: Précis de littérature française du Moyen Age. Daniel Poirion (éd.). Paris, Presses Universitaires de France, p. 128-155.

ZINK, Michel ; WIND, Bartina H. 1992: «Courtoisie». In: Dictionnaire des lettres françaises. Le Moyen Age. Geneviève Hasenohr, Michel Zink (éds). Paris, Fayard, p. 334-342.

\section{Note bio-bibliographique}

Anna Gęsicka, historienne de la littérature française, médiéviste, maître de conférences à l'Université Nicolas Copernic de Torun. Doctorat 2003 : Stéréotype de la vieillesse dans la poésie française du $X V^{e}$ siècle. Habilitation 2014: Między wola a niewola. Problem wyboru w krótkich narracjach francuskiej literatury dwornej XII i XIII wieku [Entre volonté et dépendance. Le problème du choix dans les brèves narrations courtoises de la littérature française des XII ${ }^{\circ}$ et XIII ${ }^{e}$ siècles], Wydawnictwo Naukowe Uniwersytetu Mikołaja Kopernika, Toruń 2014, 375 p. Monographie en préparation: Jeux intertextuels. Les fabliaux médiévaux sous la plume de Jean Ott (XX siècle).

Principaux domaines de recherches: la vieillesse et la mort dans la littérature médiévale (poésie et narrations); la question de la volonté et du choix dans les narrations médiévales; l'érotisme et la sexualité dans la littérature et la culture de la France médiévale; l'intertextualité et le médiévalisme.

ages@umk.pl 